\title{
GMC Origins and Turbulent Motions in Spiral and Dwarf Galaxies
}

\author{
Bruce G. Elmegreen
}

\begin{abstract}
IBM Research Division, T.J. Watson Research Center, Yorktown Heights, NY USA
\end{abstract}
email: bge@us.ibm.com

\begin{abstract}
CO clouds can be non-self-gravitating in high pressure environments, while most should be strongly self-gravitating at low metallicities and ambient pressures. In the LMC, which is HI-rich, GMC formation and destruction should generally include molecule formation and destruction. In M51, which is CO-rich, GMCs grow by coalescence. The Milky Way is between these two situations. In all cases, large clouds form by accretion of gas and smaller clouds independently of the presence of molecules. GMCs in the Milky Way are analogous to dust lanes and spurs in other galaxies. The virial parameter $\alpha$ usually decreases monotonically with increasing cloud mass in surveys, which implies that small scale structure is formed by turbulence. Hierarchies of sequences with decreasing $\alpha$ should be present in cloud complexes from sub-solar masses up to the ambient Jeans mass $\left(10^{7} M_{\odot}\right)$.
\end{abstract}

Keywords. stars: formation — ISM: clouds — galaxies: spiral — galaxies: dwarf

\section{Introduction: the Molecular Transition}

Star-forming gas is usually traced by molecular line emission from CO. $\mathrm{H}_{2}$ can be extensive without CO (e.g., Barriault et al. 2010, Lee et al. 2012), although pure- $\mathrm{H}_{2}$ is still apparently confined to diffuse regions locally, not dense self-gravitating cores. For this reason, CO line emission is fundamental for studying dense cloud structures, and it is important to consider the different types of clouds that emit CO. Such clouds need not be self-gravitating, for example. The processes that assemble clouds are evident from observations, although CO-cloud lifetimes are uncertain.

Local ${ }^{13} \mathrm{CO}$ clouds have a minimum extinction in optical bands of around 1.5 magnitudes (Pineda et al. 2008). This corresponds to a mass column density of $\Sigma_{\mathrm{CO}} \sim 30 M_{\odot}$ $\mathrm{pc}^{-2}$ with solar abundances, and to a self-gravitating pressure of $P_{\mathrm{CO}} \sim(\pi / 2) G \Sigma_{\mathrm{CO}}^{2}=$ $3 \times 10^{4} \mathrm{k}_{\mathrm{B}} \mathrm{K} \mathrm{cm}^{-3}$. This is the maximum boundary pressure for a marginally-CO cloud to be strongly self-gravitating. Regions with higher pressures can have CO clouds that are not strongly self-gravitating ("pressure-bound" clouds). The threshold pressure is higher than the average thermal pressure in the local ISM, so local CO clouds are selfgravitating. Examples of $\mathrm{CO}$ clouds close to the extinction threshold for CO-formation are the translucent clouds found by Blitz et al. (1984) and studied more recently by Barriault et al. (2010). They contain CO in denser regions but are generally pressure-bound. $\mathrm{CO}$ in local diffuse clouds was measured in absorption by Federman et al. (1980).

At lower metallicity, 1.5 magnitudes of visual extinction corresponds to a higher mass column density, in inverse proportion to the dust-to-gas ratio. Then the ambient pressure minimum for pressure-bound CO clouds is higher in proportion to the square of the gasto-dust ratio. Clouds at the minimum extinction for $\mathrm{CO}$ are therefore more likely to be strongly self-gravitating at lower metallicity, as in dwarf galaxies. The ambient pressure is also lower in dwarfs, making any clouds that appear with $\mathrm{CO}$ even more self-gravitating. 
Molecular hydrogen appears in clouds that have much lower column densities than CO-bearing clouds. In the solar neighborhood, the threshold for $\mathrm{H}_{2}$ occurs at about $A_{V} \sim 0.3 \mathrm{mag}$ (Spitzer \& Jenkins 1975), where the mass column density is $\Sigma_{\text {gas }}=6 M_{\odot}$ $\mathrm{pc}^{-2}$ (including He and heavy elements). This threshold is half the commonly observed $\mathrm{HI}-\mathrm{H}_{2}$ transition in galaxies (Shaya \& Federman 1987, Bigiel et al. 2008). The maximum pressure for strongly self-gravitating, barely- $H_{2}$ clouds is $P_{\mathrm{H} 2} \sim(\pi / 2) G \Sigma_{\mathrm{H} 2}^{2}=1200 k_{\mathrm{B}}$ $\mathrm{K} \mathrm{cm}^{-3}$, which is $\sim 1 / 3$ the ambient thermal pressure in the solar neighborhood. Thus $\mathrm{H}_{2}$-rich and CO-poor clouds in the solar neighborhood are diffuse.

The actual appearance of CO in emission also depends on the density, especially at low column density near the CO threshold where the CO lines are optically thin. Krumholz (2011) shows that $\mathrm{CO}(1-0)$ is not fully excited until the $\mathrm{H}_{2}$ density reaches $\sim 10^{3} \mathrm{~cm}^{-3}$. A region with the threshold ${ }^{13} \mathrm{CO}$ column density of $A_{V} \sim 1.5 \mathrm{mag}$ and an average $\mathrm{H}_{2}$ density of $10^{3} \mathrm{~cm}^{-3}$ is only $0.45 \mathrm{pc}$ thick. Such a region, as a cubical volume, actually has a mass $\left(6 M_{\odot}\right)$ that exceeds the thermal Jeans mass $\left(2.9 M_{\odot}\right)$ at $10 \mathrm{~K}$ and the same density. Note that with this density, the internal pressure is comparable to the threshold for CO given above and larger than the ambient thermal pressure, so fully excited CO clouds are strongly self-gravitating at the local ambient thermal pressure. A corollary to this statement is that local diffuse clouds close to the CO-formation threshold should be only marginally excited in $\mathrm{CO}$ and difficult to observe in emission. In higher pressure environments, such as spiral arm dust lanes and inner regions of galaxies, diffuse clouds (i.e., those at the local pressure) close to the ${ }^{13} \mathrm{CO}$-formation threshold should be well excited and more easily observed (Elmegreen 1993, Shetty et al. 2012). For this reason, we expect high pressure environments to contain observable diffuse CO, i.e., CO clouds that are not strongly self-gravitating.

The transition to $\mathrm{H}_{2}$ depends on both density and column density because $\mathrm{H}_{2}$ selfshields by absorption in saturated lines. Equilibrium is achieved when the formation rate of $\mathrm{H}_{2}$ on grains (a density-squared process integrated over the path length of the shielding layer) equals the destruction rate from external radiation. The threshold column density therefore scales inversely with density for a given radiation field and metallicity. Krumholz et al. (2008) write this as a dust optical depth for the shielding layer $\tau=n \sigma_{d} z=\ln (1+\chi)$ where $\chi \propto E / n Z$ for density $n$, dust cross section $\sigma_{d}$, shielding length $z$, radiation field $E$, and metallicity $Z$, which enters into the molecule formation rate on grain surfaces. For clouds shielded mostly by $\mathrm{H}_{2}$ self-absorption, $\chi<1, \ln (1+\chi) \sim \chi$ and the shielding column density $n z$ is proportional to $1 / n$ (times $E / Z$ ). For clouds shielded by dust, $n z$ increases with $1 / n$ logarithmically. Sufficiently massive clouds in a given radiation field can be atomic and self-gravitating because they have low densities in spite of their high column densities (Elmegreen \& Elmegreen 1987). The low densities result from high velocity dispersions at a fixed ambient pressure. This is a manifestation of Larson's scaling law between density and mass: at a given pressure for a virialized cloud, the density scales inversely with the square root of the mass. Pineda et al. (2008) found a higher density threshold for CO formation in higher dispersion clouds because of this opacity effect.

\section{Cloud Formation}

These relations between pressure, metallicity, self-gravity, and the presence of molecules help us to understand the various ways in which molecular clouds form. Cloud formation or assembly by itself is the result of converging flows that bring together ambient material and other clouds to make new, larger clouds. The flows can be initiated by self-gravity, turbulence, expanding shells, spiral wave shocks, and other dynamical processes. When 
the ISM metallicity or pressure are low, as in the LMC, the ISM is mostly atomic and molecular clouds form by dynamical flows accompanied by a transition from atoms to molecules. The molecules are in the self-gravitating parts of the cloud. When star formation tears these clouds apart, they mostly revert back into atomic gas. The result is a low molecular fraction and spotty CO distribution, as observed in the LMC (Wong et al. 2011). When the metallicity or pressure are high, as in M51 (Koda et al. 2009), or the radiation field is very low, then the ISM is mostly molecular. Even a high fraction of diffuse clouds could be molecular, and the most massive self-gravitating clouds (at the ambient Jeans mass of $\sim 10^{7} M_{\odot}$ ) can be molecular too. These are the giant molecular associations in M51 (Vogel et al. 1988), which appear in M51's spiral arms like similar-mass atomic clouds in the Milky Way (Elmegreen \& Elmegreen 1987). Cloud formation in a molecule-rich ISM is still by dynamical flows, but the flows themselves are molecular. Then we can speak of GMC formation as a process of GMC collisions, although physically is it probably the same as in an atom-rich medium. In both cases, a clumpy, turbulent medium converges to make giant clouds and strongly self-gravitating cloud complexes.

The state of molecular gas in the Milky Way is somewhat between the extremes of the LMC and M51: it is largely atomic near the solar radius and beyond, and half-way molecular in the inner disk. There is apparently a transition from mostly atomic gas in the interarm regions and some parts of spiral arms to mostly molecular in the densest parts of the arms, which are presumably spiral shocks, implying an HI-CO transition during cloud build-up followed by some $\mathrm{CO}$ destruction as gas flows out of the arms. Because of the relatively high spiral arm pressure, much of the CO in the spiral shocks can be in the form of diffuse clouds. Most of the Milky Way's GMCs are in the cores

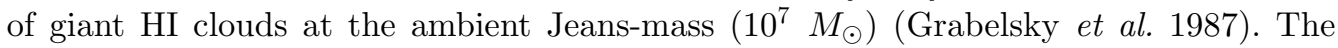
average densities of these clouds are low $\left(\sim 9 \mathrm{~cm}^{-3}\right.$; Elmegreen \& Elmegreen 1987), and they should be disrupted by shear and tidal forces when they emerge from the arms.

The association of massive Milky Way CO clouds with spiral arm shocks is apparent in the distribution of CO from Dame et al. (2001) combined with kinematic models of the spiral arm gas flow (Bissantz et al. 2003). The concentration of CO in the arms is apparent in the face-on view presented by Englmaier et al. (2011). These CO clouds would appear as dust lanes in other galaxies.

\section{The Onset of Star Formation in GMCs}

Surveys of molecular clouds usually show a lack of self-gravity on small scales, with increasing self-gravity for more massive clouds. This trend is usually evident in a plot of the dimensionless virial parameter $\alpha\left(=M_{\text {virial }} / M\right)$ versus the cloud mass. $\alpha$ is high for non-self-gravitating clouds. In surveys, it is usually high for low mass clouds and decreases with increasing mass (Dawson et al. 2008, Lada et al. 2008, Schlingman et al. 2011, Barnes et al. 2011, Belloche et al. 2011, Giannini et al. 2012).

The implication of this trend is that small clouds in homogeneous surveys form by nongravitational processes, which are most likely dominated by ram-pressure driven convergence in a supersonically turbulent medium. Only the largest scales are self-gravitating.

Hirota et al. (2011) show the change in $\alpha$ for GMCs that enter a spiral arm in IC 342. Just before entering, the $\mathrm{CO}$ clouds have high $\alpha$ and no star formation. Inside the arm, cloud velocity dispersions and $\alpha$ 's drop, and star formation begins. Spiral arm compression appears to enhance turbulent dissipation and self-gravity in the clouds.

In a more comprehensive survey, which includes various molecules with different density sensitivities, $\alpha$ would not necessarily show a monotonic change with mass. Most likely, 
clouds are a nested hierarchy of self-gravitating and non-self-gravitating structures, with either occurring inside the other. For example, self-gravity in the ambient ISM can make a Jeans-mass cloud at $10^{7} M \odot$, and this cloud can fragment by turbulence compression (producing an $\alpha$ sequence). The massive fragments then become more self-gravitating after energy dissipation, forming GMCs with locally low $\alpha$. These GMCs simultaneously fragment more by turbulence, producing another $\alpha$ sequence inside of them on smaller scales. Turbulent fragmentation stops when the sonic scale is reached. If the sonic scale, which is initially high- $\alpha$, becomes self-gravitating, perhaps after mass accretion, then the associated structure can form a star. All of this happens in a dynamically collapsing, turbulent region, so $\alpha$ is likely to contain shearing and convergent or divergent motions.

We note the important difference between the gravitational time $\left([G \rho]^{-1 / 2}\right)$ and the crossing time $(R / \Delta V)$. The ratio of these two times is the square root of $\alpha$. For small regions inside a virialized cloud, where $\alpha$ is large, the turbulent crossing time is shorter than the gravitational time and turbulent motions dominate. To form a star, such a region has to lower its $\alpha$ by energy dissipation and mass accretion before the ambient flow disrupts it. If the small region is non-isotropic, like a filament, then it can have effectively low $\alpha$ in some directions and high $\alpha$ in other directions. Filaments form stars by accretion along the axis on a gravitational time, which is longer than the crossing time on the minor axis.

\section{References}

Barnes, P. J., et al. 2011, ApJS, 196, 12

Barriault, L., et al. 2010, MNRAS, 406, 2713

Belloche, A., et al. 2011, A\&A, 535, 2

Bigiel, F., et al. 2008, AJ, 136, 2846

Bissantz, N., Englmaier, P., \& Gerhard, O. 2003, MNRAS, 340, 949

Blitz, L., Magnani, L., \& Mundy, L. 1984, ApJ, 282, L9

Dame, T. M., Hartmann, D., \& Thaddeus, P. 2001, ApJ, 547, 792

Dawson, J. R., et al. 2008, MNRAS, 387, 31

Elmegreen, B. G. 1993, ApJ, 411, 170

Elmegreen, B. G. \& Elmegreen, D. M. 1987, AJ, 320, 182

Englmaier, P., Pohl, M., \& Bissantz, N. 2011, MmSAI, 18, 199

Federman, S. R., Glassgold, A. E., Jenkins, E. B., \& Shaya, E. J. 1980, ApJ, 242, 545

Giannini, T., et al. 2012, A\&3A, 539, 156

Grabelsky, D. A., Cohen, R. S., Bronfman, L., Thaddeus, P., \& May, J. 1987, ApJ, 315, 122

Hirota, A., Kuno, N., Sato, N., Nakanishi, H., Tosaki, T., \& Sorai, K. 2011, ApJ, 737, 40

Koda, J., et al. 2009, ApJ, 700, L132

Krumholz, M. R., McKee, C. F., \& Tumlinson, J. 2008, ApJ, 689, 865

Krumholz, M. R. 2011, in XVth Special Course of the National Observatory of Rio de Janeiro, arXiv: 1101.5172

Lada, C. J., Muench, A. A., Rathborne, J., Alves, J. F., \& Lombardi, M. 2011, ApJ, 672, 410

Lee, M.-Y., et al. 2012, ApJ, 748, 75

Pineda, J. E., Caselli, P., \& Goodman, A. A. 2008, ApJ, 679, 481

Schlingman, W. M., et al. 2011, ApJS, 195, 14

Shaya, E. J. \& Federman, S. R. 1987, ApJ, 319, 76

Shetty, R., Kelly, B. C., \& Bigiel, F. 2012, preprint

Spitzer, L., Jr. \& Jenkins, E. B. 1975, ARA \& A, 13, 133

Vogel, S. N., Kulkarni, S. R., \& Scoville, N. Z. 1988, Nature, 334, 402

Wong, T., et al. 2011, ApJS, 197, 16 